\title{
Congenital Heart Anomalies in Foetuses: Therapeutic Interventions
}

\section{Dinarević SM*}

Committee for Cardiovascular Pathology, Academy of Sciences and Arts, Sarajevo, Bosnia and Herzegovina

*Corresponding author: Dinarević SM, Committee for Cardiovascular Pathology, Academy of Sciences and Arts, Sarajevo, Bosnia and Herzegovina, Tel: +38733560700; E-mail: senka@bih.net.ba

Received: November 28, 2018; Accepted: December 13, 2018; Published: December 19, 2018

Copyright: () 2018 Dinarević SM. This is an open-access article distributed under the terms of the creative commons attribution license, which permits unrestricted use, distribution, and reproduction in any medium, provided the original author and source are credited.

\begin{abstract}
In the last two decades there has been an enormous step forward in the diagnosis and therapeutic interventions (TI) in foetuses with congenital heart anomalies. Prenatal diagnosis and therapy not only require many professional skills, but entails many ethical and legislative consequences, causing a critical attitude. Therefore, the experts dealing with fetology are obliged to present their results, no matter what they are, because only in this way the contribution to the development and proper understanding of the concept could be made. Understanding the natural history of the malformation and the continual ability to refine patient selection are critical for possible $\mathrm{TI}$.
\end{abstract}

Keywords: Foetus; Therapeutic; Intervention

\section{Introduction}

Thanks to advances in imaging techniques, multidisciplinary teamwork, acquired experience and interest in foetal medicine, in the last two decades there has been an enormous step forward in the diagnosis and therapeutic interventions (TI) in foetuses with congenital heart anomalies (CHA). Prenatal diagnosis and therapy not only require many professional skills, but entails many ethical and legislative consequences, causing a critical attitude. So, the experts dealing with fetology are obliged to present their results, no matter what they are, because only in this way the contribution to the development and proper understanding of the concept could be made. Understanding the natural history of the malformation and the continual ability to refine patient selection, are critical [1]. Foetal cardiac intervention offers the potential to alter in utero anatomy and physiology so that it either is lifesaving to the foetus or results in an improved state at birth that leads to reduction in short- or long-term morbidity or mortality. Medical and interventional treatments for select diseases and strategies for delivery room care by well-trained multidisciplinary team with sophisticated equipment are required for improving the technical success rate and post-interventional outcomes.

The first drawing in the human history of "foetus in utero" was given by Leonardo da Vinci back in 1507 and hints of modern imaging techniques such as MRI and 3D computer modelling. The development of $2 \mathrm{D}$ as well as $3 \mathrm{D}$ echocardiographic techniques allows possibilities to visualize and diagnose CHA of foetuses and to assist in successful early intervention of certain CHA. Pregnancy where the expected potential problems or abnormalities of the foetus are expected, requires the involvement of a multidisciplinary team that will take care of mother and child. In addition to the formation of multidisciplinary teams that are aimed at protecting the mother and foetus, there is a tendency to institutionalize the pathology associated with the foetus and established the so-called: foetal centres. The study of foetal physiology and foetal condition become the main preoccupation of these centres, focused primarily on antenatal diagnosis and therapy, as well as the development of a new model, called MADAM model (Multidisciplinary Antenatal Diagnosis and Management). Pregnancy in which a foetal team would be included are screened from the population of pregnant women in which are found: ultrasound foetal abnormality, where there are proven chromosomal abnormalities, pregnancy with risk factors for hereditary metabolic or genetic disease, as well as pregnant women who were exposed to teratogenic causes during pregnancy. In the plans for the development of foetal medicine also is included a palliative care of foetus in order to prolong the life of the foetus that has identified anomalies, which is another big challenge, but undoubtedly philosophical and ethical question. Caring for the health of the foetus should begin in the family even before conception, and that the trend in the world of family care which is entrusted into the hands of family doctors, the American Academy of Paediatrics made a recommendation for family doctors in terms of protecting and promoting the health of the foetus. The foetus health and the health of the child is conditioned by the health of both parents, their emotional and social health, social conditions in which the family lives, as well as the attitude towards the child. The mother's right is to decide on the health of the unborn child. The right of the unborn child is to have access to all therapeutic opportunities and the right of doctors is to use their knowledge and skills to help the unborn child as a patient, they're all still moral and ethical problems that follow in the next period of medical research and good medical practice.

\section{Literature Review}

Cardiac lesions that are amenable to foetal intervention are distinctive in the way that they can progress rapidly from mild to severe during gestation such that there is significant irreversible myocardial damage and chamber, valve, or vessel hypoplasia at the time of birth. In this unique group of defects, there is commonly a time-limited window of opportunity to intervene when deleterious effects on cardiac growth and function are deemed to be potentially reversible. However, for some anomalies in which an alteration in prenatal natural history for the better is possible and for those with extremely poor outcome, foetal cardiac intervention may be the best course of action and is a reasonable therapeutic option.

Several developmental pathways can result in Hypoplastic left heart syndrome (HLHS), most of which are not amenable to foetal cardiac intervention. The lesion that has been the main focus of foetal cardiac 
intervention over the past 2 decades is severe aortic stenosis (AS) in early gestation and mid-gestation, which has been shown to evolve into HLHS at birth [2]. As with evolving HLHS starts out with the cardiac chambers normally formed and most often with normal function in the first and even second trimester's [3]. As the stenosis becomes more severe, progressive left ventricle (LV) dysfunction develops, and flow reversal at the foramen ovale and aortic arch eventually occurs such that blood is diverted away from the left heart. This, along with myocardial and valvar damage and hypoplasia, results in HLHS at birth. Despite the relatively small numbers, insight into the natural and unnatural histories of this lesion has been gained that has enabled more accurate selection of patients who might attain benefit from this intervention. Selection guidelines have been described and are reasonable to use for assistance in determining which foetuses are likely to benefit. First, the anatomy must be favourable in that there is AS and not atresia with evidence for antegrade flow across the aortic valve on Doppler assessment of the valve. In addition, there should be no or minimal sub valvar left ventricular outflow obstruction. Second, there should be strong evidence for the process of evolving HLHS based on the presence of depressed LV function and flow abnormalities determined by foetal echocardiogram.

Factors predicting a favourable outcome for 2-ventricle repair include: a LV long-axis $\mathrm{z}$ score of -2 , the LV being able to generate a pressure of at least $10 \mathrm{~mm} \mathrm{Hg}$ across the aortic valve or a $15-\mathrm{mm} \mathrm{Hg}$ mitral regurgitate jet, and a mitral valve (MV) diameter $\mathrm{z}$ score of -3 . The larger the LV and MV are, and the greater the ability are for the LV to generate reasonable pressure, the greater the likelihood is of a successful ultimate biventricular circulation [4]. Before the procedure, extensive family counselling should detail the risks of the procedure to mother and foetus and lay out the expected clinical course for those who undergo intervention to those who choose more standard management. Essential in the treatment of evolving HLHS is postnatal management of the infant. The neonatal and ongoing management of these patients requires insight and experience with the natural and unnatural histories of the borderline left heart. A key element of achieving a biventricular circulation in these patients is the postnatal decision making, including the use of specialized interventional catheterization procedures and surgery.

Foetal intervention alone is unlikely to be adequate therapy to achieve a biventricular circulation in all candidates; therefore, delivery and management at a specialized congenital heart centre are recommended. It is important to appreciate the potential benefits and promise of foetal cardiac catheter intervention for critical AS evolving into HLHS by possibly creating a postnatal 2-ventricle system, the long-term benefits and outcomes of this procedure are unknown. Although outcomes for HLHS after the Fontan operation and the limitations of this strategy are relatively clear, the foetus undergoing a cardiac catheter intervention for AS may be at future risk for multiple operations, valve replacements, ventricular dysfunction, and possibly pulmonary hypertension within the context of a borderline-size small left ventricle. Families should be counselled about these concerns and about the lack of data on long-term outcomes. Comparative analysis of these alternative strategies through careful investigational efforts is warranted.

The foetus with HLHS and highly restrictive or intact atrial septum, is stable in utero, although there is likely continuing damage to the pulmonary vasculature and lung parenchyma. Typically, the new-born becomes critically ill immediately after birth when blood is unable to exit the left atrium/LA and succumbs to a combination of hypoxia, acidosis, and pulmonary oedema. If such a patient goes undiagnosed prenatally and survival is unlikely. If diagnosed prenatally, a wellplanned delivery with urgent transfer to the catheterization laboratory can be arranged for decompression of the left atrium by balloon dilation or stent dilation of the atrial septum; however, outcomes remain poor [5]. Theoretically, some of the devastating effects on the lungs and vasculature may be reversible if an intervention can be performed at a critical point in gestation. Because some level of restriction at the atrial septum is typical in HLHS, identifying those in whom a critical degree of atrial obstruction is present is essential in identifying candidates who will benefit from foetal intervention. a) Foetal Doppler assessment of pulmonary venous flow patterns: the greater prominence of flow reversal during atrial contraction reflecting greater restriction [6]. b) Assessment of pulmonary arterial impedance through Doppler imaging during maternal hyperoxygenation can test for pulmonary vasoreactivity in the foetus with HLHS. Either or both assessments are reasonable to obtain for determination if foetal intervention may be beneficial. Several techniques used to open the atrial septum have been reported. Questions concerning the most effective technique for opening the atrial septum in utero, including balloon atrial septoplasty versus stent placement, in addition to the optimal timing to perform the procedure to mitigate against the development of pulmonary vasculopathy, remain unanswered [7]. However, given the significant mortality and morbidity of HLHS with a restrictive or intact atrial septum, foetal intervention may be reasonable to perform in this disease, not only to stabilize the patient in the immediate postnatal period but also to potentially prevent or reverse the damage to the lungs and vasculature.

Incompetence of the mitral valve is typically attributable to a mitral valve/MV arcade with combined stenosis and insufficiency. Severe mitral regurgitation/MR leads to LA dilatation with a restrictive or intact atrial septum. MR results in a dilated LV, a dilated LA, and secondary closure of the foramen ovale. Severe dilatation of left-sided structures may compress the right side, leading to hydrops, which, if present, is most often lethal. Foetal cardiac intervention may be considered to open the aortic valve and to promote forward flow [8]; however, aortic regurgitation after the procedure may complicate the physiology. Opening of the atrial septum with the goal of decompressing the LA and improving filling of the right side has also been proposed [9] and may be considered. LV dysfunction and MV disease may still prevent the use of the LV for a biventricular repair, and a single-ventricle strategy may still be necessary after birth.

\section{Discussion and Conclusion}

Only a small subset of foetuses with pulmonary atresia and intact ventricular septum - (PA/IVS) should be considered candidates for foetal cardiac intervention. The goal is to prevent the need for singleventricle palliation after birth. Intervention in this lesion is controversial because there are limited studies describing the natural history and foetal predictors of postnatal outcome [10]. The threshold for right ventricle (RV) inadequacy and nonviability as a pulmonary ventricle is much higher than is the value for inadequacy of the $L V$ as a systemic ventricle. Even in very small RV, as long as the tricuspid valve (TV) is of an appropriate size, continued rehabilitation of the RV can take place through staged surgical palliation after birth, which can result in successful achievement of a biventricular repair. In addition to promoting RV growth and avoiding a single-ventricle palliation, another possible indication for intervention in right-sided disease is in the group with PA/IVS, severe tricuspid regurgitation (TR), and 
Citation: Dinarevic SM (2018) Congenital Heart Anomalies in Foetuses: Therapeutic Interventions. J Cardiovasc Dis Diagn 6: 349. doi: 10.4172/2329-9517.1000349

Page 3 of 3

hydrops in whom impending foetal demise is anticipated [11]. In such circumstances, prenatal intervention may be lifesaving to the foetus. The technique for intervention in PA/IVS is more difficult than it is for the aortic valve given that the RV cavity is commonly small, hypertrophied, and located behind the sternum. Future directions of foetal cardiac interventions are a combination of nonsurgical cardiac therapy with minimally invasive surgical techniques.

\section{Conflicts of Interest}

There are no conflicts of interest for the present study.

\section{References}

1. Dinarević S (2015) Fetalna medicina kod Leonarda da Vinci do danas: Scientific International Symposium, Academy of Sciences and Arts of Bosnia and Herzegovina, special edition, Department of Medical Sciences: ISBN: 9789926 -410-00-1 46: 7-15.

2. Mäkikallio K, McElhinney DB, Levine JC, Marx GR, Colan SD, et al. (2006) Clinical perspective. Circulation 113: 1401-1405.

3. Hornberger LK, Sanders SP, Rein AJ, Spevak PJ, Parness IA, et al. (1995) Left heart obstructive lesions and left ventricular growth in the midtrimester fetus: A longitudinal study. Circulation 92: 1531-1538.

4. McElhinney DB, Marshall AC, Wilkins-Haug LE, Brown DW, Benson CB, et al. (2009) Predictors of technical success and postnatal biventricular outcome after in utero aortic valvuloplasty for aortic stenosis with evolving hypoplastic left heart syndrome. Circulation 120: 1482-1490.
5. Vlahos AP, Lock JE, McElhinney DB, Velde ME (2004) Hypoplastic left heart syndrome with intact or highly restrictive atrial septum: Outcome after neonatal transcatheter atrial septostomy. Circulation 109: 2326-2330.

6. Divanović A, Hor K, Cnota J, Hirsch R, Kinsel-Ziter M, et al. (2011) Prediction and perinatal management of severely restrictive atrial septum in fetuses with critical left heart obstruction: Clinical experience using pulmonary venous Doppler analysis. J Thorac Cardiovasc Surg 141: 988-994.

7. Marshall AC, Levine J, Morash D, Silva V, Lock JE, et al. (2008) Results of in utero atrial septoplasty in fetuses with hypoplastic left heart syndrome. Prenat Diagn 28: 1023-1028.

8. Vogel M, McElhinney DB, Wilkins-Haug LE, Marshall AC, Benson CB, et al. (2011) Aortic stenosis and severe mitral regurgitation in the fetus resulting in giant left atrium and hydrops: Pathophysiology, outcomes, and preliminary experience with pre-natal cardiac intervention. J Am Coll Cardiol 57: 348-355.

9. Rogers LS, Peterson AL, Gaynor JW, Rome JJ, Weinberg PM, et al. (2011) Mitral valve dysplasia syndrome: A unique form of left-sided heart disease. J Thorac Cardiovasc Surg 142: 1381-1387.

10. Roman KS, Fouron JC, Nii M, Smallhorn JF, Chaturvedi R, et al. (2007) Determinants of outcome in fetal pulmonary valve stenosis or atresia with intact ventricular septum. Am J Cardiol 99: 699-703.

11. Tulzer G, Arzt W, Franklin RC, Loughna PV, Mair R, et al. (2002) Fetal pulmonary valvuloplasty for critical pulmonary stenosis or atresia with intact septum. Lancet 360: 1567-1568. 